\title{
Cerebral Networks of Interfacial Water: Analogues of the Neural Correlates of Consciousness in a Synthetic Three-Shell Realistic Head Model
}

\author{
Nicolas Rouleau, Michael Persinger* \\ Behavioural Neuroscience Laboratory, Laurentian University, Sudbury, Canada \\ Email: "
}

Received 27 August 2014; revised 23 September 2014; accepted 17 October 2014

Copyright (C) 2014 by authors and Scientific Research Publishing Inc.

This work is licensed under the Creative Commons Attribution International License (CC BY). http://creativecommons.org/licenses/by/4.0/

(c) (i) Open Access

\begin{abstract}
The physical properties of water, particularly the nature of interfacial water and pH shifts associated with dynamics of the hydronium ion near any surface, may be a primary source of the complex electromagnetic patterns frequently correlated with consciousness. Effectively all of the major correlates of consciousness, including the $40 \mathrm{~Hz}$ and $8 \mathrm{~Hz}$ coupling between the cerebral cortices and hippocampal formation, can be accommodated by the properties of water within a specific-shaped volume exposed to a magnetic field. In the present study, quantitative electroencephalographic activity was measured from an experimental simulation of the human head constructed using conductive dough whose $\mathrm{pH}$ could be changed systematically. Spectral analyses of electrical potentials generated over the regions equivalent to the left and right temporal lobes in humans exhibited patterns characteristic of Schumann Resonance. This fundamental and its harmonics are generated within the earth-ionospheric cavity with intensities similar to the volumetric intracerebral magnetic $(\sim 2 \mathrm{pT})$ and electric field $\left(\sim 6 \times 10^{-1} \mathrm{~V} \cdot \mathrm{m}^{-1}\right)$ strengths. The power densities for specific $\mathrm{pH}$ values were moderately correlated with those obtained from normal human brains for the fundamental (first) and second harmonic for the level simulating the cerebral cortices. Calculations indicated that the effective $\mathrm{pH}$ would be similar to that encountered within a single layer of protons near the plasma membrane surface. These results reiterate recent measurements in a large population of human brains showing the superimposition of Schumann power densities in QEEG data and indicate that intrinsic features of proton densities within cerebral water may be a fundamental basis to consciousness that can be simulated experimentally.
\end{abstract}

\section{Keywords}

Interfacial Water, Hydronium Ion, Electromagnetic Consciousness, Cerebral Water, Schumann

\footnotetext{
${ }^{*}$ Corresponding author.
}

How to cite this paper: Rouleau, N. and Persinger, M. (2014) Cerebral Networks of Interfacial Water: Analogues of the Neural Correlates of Consciousness in a Synthetic Three-Shell Realistic Head Model. Journal of Signal and Information Processing, 5, 143-154. http://dx.doi.org/10.4236/jsip.2014.54017 


\section{Resonance, QEEG}

\section{Introduction}

Neuroanatomical organization of a sufficient complexity is thought to be a necessary condition of consciousness [1] wherein neural networks form and the resulting structure dictates the function of the organ [2]. Neurons, composed mainly of water and proteins [3], communicate by means of electrochemical signalling with direction and magnitude which result in cognitive states and behaviours. However, as the convolutions which constitute the structure of the brain became increasingly complex, so did the intervening negative spaces-including the extracellular environment. Studying consciousness exclusively within cells is problematic when considering that the medium between cells is primarily composed of water with ions [3] and that solutions such as these are able to store and release electromagnetic energy [4]. The implication is that the proteinaceous structure of the brain itself is an epiphenomenon which operates simultaneous to a covert system where neurons express activity from the local environment in an integrated network of aqueous boundary conditions.

That the relatively stable microstructual patterns, at the level of the synapse, function to maintain the temporal consistency of the conditions that correlate with consciousness, is a viable, alternative possibility. Water molecules adjacent to a surface exhibit all of the major properties attributed to the plasma cell membrane. Interfacial water, compared to bulk water, displays about ten times the viscosity. The resulting exclusion zone is associated with a narrow zone of protons. The generated potential difference ranges in the order of $100 \mathrm{mV}$ which is the same order of magnitude as the resting membrane potential traditionally attributed to the disparity in concentration of cations and anions across the membrane space. Within the exclusion zone generated by ordinary water adjacent to a surface, electromagnetic fields can be retained for protracted periods of time [5].

Water displays universal properties derived from fundamental physical parameters. For example its diffusivity $\left(0.8 \times 10^{-7} \mathrm{~m}^{2} \cdot \mathrm{s}^{-1}\right)$ is obtained by dividing the magnetic moment of a proton $\left(1.41 \times 10^{-26} \mathrm{~A} \cdot \mathrm{m}^{2}\right)$ by the unit charge $\left(1.6 \times 10^{-19} \mathrm{~A} \cdot \mathrm{s}\right)$. When this quotient is multiplied by the typical viscosity of water $\left(1.0 \times 10^{-3} \mathrm{~kg} \cdot \mathrm{m}^{-1} \cdot \mathrm{s}^{-1}\right)$ at $20^{\circ} \mathrm{C}$ the resulting force is $8.8 \times 10^{-11} \mathrm{~kg} \cdot \mathrm{m} \cdot \mathrm{s}^{-2}$ [5]. When applied across the width of two O-H bonds $(1.92 \times$ $10^{-10} \mathrm{~m}$ ) the energy is about $1.7 \times 10^{-20} \mathrm{~J}$. This value is within the measurement error of the energy for the second shell hydrogen bond that reflects proton mobility in water [6]. We think it is relevant that $\sim 1.7 \times 10^{-20} \mathrm{~J}$ is within the range of energy associated with effects of a typical neuronal action potential upon a unit charge and the energy required to stack one nucleotide onto an RNA sequence [7]. In other words, the most fundamental increment of energy associated with the neuronal correlate of consciousness and cognition, the action potential, and energy required to add different nucleotides, the determinant of the protein patterns of memory, is the same value as that associated with the movements of protons through water.

The proton is a primary candidate to mediate information within brain space. According to DeCoursey [6] the proton is unique among cations in being interchangeable with the protons that form water. There is a five-fold higher conductivity for $\mathrm{H}^{+}$in water compared to other cations such as $\mathrm{K}^{+}$and there are more proton channels than the sum of channels from all other ions combined. Even when one considers the numbers of free protons within the hydronium atom is about $40 \mathrm{nM}$ (compared to the $110 \mathrm{M}$ of hydrogen in water), the numbers become significant when one appreciates that most of the brain volume is water. During a $100 \mathrm{pA}$ outward $\mathrm{H}^{+}$current between $10^{8}$ and $10^{9} \mathrm{H}^{+}$leave the typical cell. The importance and prevalence of protons and proton channels has been attributed to the evolutionary necessity of quickly dissipating the intracellular diminished $\mathrm{pH}$ associated with interactions between organic acids.

Shifts in $\mathrm{pH}$ along neuronal membranes accompany action potentials and occur as transients with intervals between 10 and $40 \mathrm{~ms}$ [8]. Highstein et al. [9] showed that protons act as neurotransmitters with shifts of $\sim 0.2$ pH near synaptic clefts. Recently Murugan et al. [10] observed that continuous exposure of spring water with ion concentrations similar to biological fluids to physiologically-patterned magnetic fields produced frequent temporal shifts of small increments in $\mathrm{pH}$ whose durations were between 20 and $40 \mathrm{~ms}$. The relevance of this increment, which is the $\sim 40 \mathrm{~Hz}$ activity associated with higher functions for cerebral activity such as consciousness, is well known. Llinas and Ribary [11] found that cohesive integrative waves displaying phase modulation moved as recursive phenomena in a rostral to caudal direction every 20 to $25 \mathrm{~ms}$. Several authors have noted the "coincidence" that the time required for bulk velocity action potentials to move through the hippocampal, tha- 
lamic, cerebral "circuits" displays durations of approximately 20 to $25 \mathrm{~ms}$. This reiterates our approach that the structure of pathways through which consciousness is mediated are arranged to ensure the specific timing of the circuit to match intrinsic features of water.

Persinger [12] proposed that the Earth's quasi-static main magnetic field is a potential medium by which all brains can be unified at a fundamental level. Although speculative and potentially interesting, the solution for the individual brain is particularly relevant. Assuming an average volume of 1350 cc which is 945 cc (70\% water), there would be $55.5 \mathrm{M}$ of water (18 cc per M). This would involve $3.16 \times 10^{25}$ water molecules. The magnetic energy from the static geomagnetic field, assuming an average of $5 \times 10^{-5} \mathrm{~T}$, would be in the order of $0.945 \times 10^{-6} \mathrm{~J}$ or $2.9 \times 10^{-32} \mathrm{~J}$ per water molecule [10]. The equivalent frequency, obtained by dividing Planck's constant $\left(6.626 \times 10^{-34} \mathrm{~J} \cdot \mathrm{s}\right)$ would be $\sim 40 \mathrm{~Hz}$. Even neural correlates of consciousness have been attributed to synchronous gamma activity [13]. The fundamental theta $(4$ to $8 \mathrm{~Hz}$ ) frequency of the hippocampal formation, the "gateway to memory", exhibits superimosed $\sim 40 \mathrm{~Hz}$ ripples that may be fundamental to interaction between cerebral cortical and parahippocampal regions [14].

The extremely-low frequency (ELF) component of the Earth's electromagnetic radiation spectrum is generated by a resonance phenomenon between its surface and the ionosphere with a fundamental operating frequency of $7.8 \mathrm{~Hz}$ [15]. Originally characterized by Schumann [16], this fundamental and its harmonics $(14.1 \mathrm{~Hz}, 20.3$ $\mathrm{Hz}, 26.4 \mathrm{~Hz}$, and $32.5 \mathrm{~Hz}$ ) are a result of global lightning discharges where the fundamental mode is determined by the circumference of the Earth. The wave forms and temporal structures associated with the Schumann frequencies are remarkably similar to the classic delta, theta, and alpha patterns attributed to cerebral cortical activity [17]. The magnetic field strength of these resonances is in the order of 1 to $3 \mathrm{pT}$, which is within the range of the operating magnetic field within the human cerebrum. If the typical $0.2 \mu \mathrm{V}$ change associated with a single ion channel is applied to a unit charge $\left(1.6 \times 10^{-19} \mathrm{~A} \cdot \mathrm{s}\right)$, the increment of energy is $0.3 \times 10^{-25} \mathrm{~J}$. The square root of this value divided by the mass of a proton is $\sim 4.5 \mathrm{~m} \cdot \mathrm{s}^{-1}$ which is the typical velocity of the rostral-caudal waves or bulk velocity of the integrated cerebral electromagnetic fields. Assuming the average circumference of a human skull to be $60 \mathrm{~cm}$, the standing wave would be between 7.5 to $7.8 \mathrm{~Hz}$. Schumann resonance frequencies are thought to influence brain activity as well as blood pressure [18] [19] and other biological processes [17].

The hippocampal formation and parahippocampal gyrus in particular are thought to be strongly coupled to the Schumann resonance [20]. The observation that the low-frequency oscillations which characterize the typical human electroencephalographic profile overlap significantly with the fundamental Schumann resonance frequency $(7.8 \mathrm{~Hz})$ and the paired harmonics might not be coincidental. Layers II and III within the entrorhinal cortices of the parahippocampal gyrus, which provides the bi-directional interactions between the cerebral cortices and hippocampal formation [21], exhibit a near-continuous subthreshold oscillation of $\sim 8 \mathrm{~Hz}$. The mean amplitude of these oscillations was $2.6( \pm 0.5) \mathrm{mV}$.

Geomagnetic storms are associated with altered human cognition and behaviour [22], suggesting some intrinsic relationship between the brain and the electromagnetic environment. Whereas it is possible that some recondite third factor is influencing both systems, experimental inductions of altered brain function using artificially generated electromagnetic fields indicate that the brain is a magnetoreceptive organ [23]. Though electromagnetic cellular interactions have been studied in detail [24], the negative brain-space of the extra-cellular environment wherein subtle physical phenomena might be occurring must be further considered as a contributing source of variance.

A negative space brain system might function by absorption and emission of electromagnetic energies as has been demonstrated in paradigms involving spring water [25], and cell cultures [26]. In both cases, externally applied stimuli were involved, suggesting that non-cerebral sources of electromagnetic energies could potentially interact with the brain by means of interaction with water. The discovery that induced, subtle shifts in $\mathrm{pH}$ within the synaptic cleft in $C$. elegans were sufficient to alter post-synaptic firing indicates the importance of proton-based neurotransmission [27]. Synapses, consisting effectively of parallel plates, operate within parameters sufficient to generate Casimir forces [28], suggesting that these interfaces are more than just transitional zones through which molecular neurotransmitters traverse. The same authors have calculated that the wavelength equivalent of the energy associated with these forces applied over $10 \mathrm{~nm}-$ a value approximating the width of the synaptic cleft and the neuronal membrane-would approximate the width of neutral hydrogen.

Together, these findings have suggested that classical mechanisms, although essential and highly reliable, do not accommodate the variety of processes observed involving neuronal signalling. Additionally, these works indicate that the proton is of central importance with regards to the functioning of the nervous system. We hy- 
pothesized that perhaps an external force or zeitgeber is ordering neural activity wherein the sum of the cellular units are necessary although insufficient to produce the resulting function in much the same way a radio receiver is necessary but insufficient in radio transmission. Whereas brain structure would be essential to brain function, the output of the brain would not be explained by cellular structures alone. As neurons would be receptive to this extra-cerebral force, it follows that the energies involved in such a process are subject to experimental measurement and quantification. Here, we present an experimental detection of what is potentially extra-cerebral information which could explain a subset of brain function normally ascribed to synchronous cellular activity.

\section{Materials \& Methods}

\subsection{Synthetic Three-Shell Realistic Head Model}

A three-shell experimental model of the human head was constructed (Figure 1) based upon theoretical models which are assumed in the dynamic neuroimaging technique of quantitative electroencephalography (QEEG) [29] [30]. The inner and outer surfaces of a plastic, replica human skull (Figure 2; Human Skull-Standard, WSP-01 by Skulls Unlimited International) were lined with an electroconductive dough (ED) in order to simulate the anatomical organization of the cortex, skull, and scalp. The replica, measuring $20 \mathrm{~cm}$ in length, $13.5 \mathrm{~cm}$ in width, and $15.5 \mathrm{~cm}$ in height, features anatomically accurate sutures, foramina, canals, fissures, condyles, and other cranial features. The organization can be described as a relative non-conductor placed between two conductors. The conductive material consists of an open-source amateur electronics recipe by Squishy Circuits. This material is typically used as an educational tool to demonstrate the principles of electrical systems (Figure 3). The dough is fabricated by mixing 237 cc of water, 355 cc of flour, 59 cc of salt, 15 cc of vegetable oil, 2 cc food coloring, and various concentrations of a proton donor (lemon juice) together in a pot over a heated surface while stirring until partially solidified. The ball of dough is then covered in 10 cc of flour, kneaded, and applied at a constant thickness of $\sim 5 \mathrm{~mm}$ in order to approximate the average human cortical thickness.

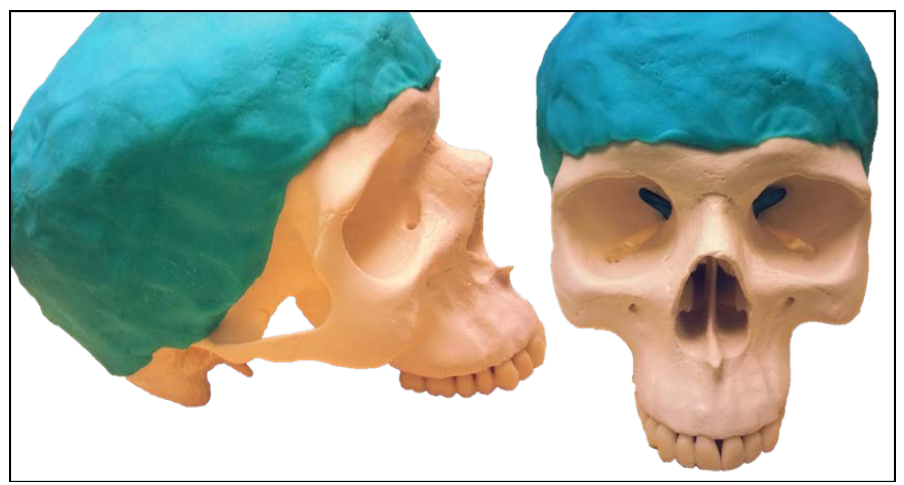

Figure 1. Synthetic three-shell realistic head model consisting of electroconductive dough which lines the inner and outer surfaces of a replica human skull. Lateral (left), rostral (right) views of the model showing the scalp layer.
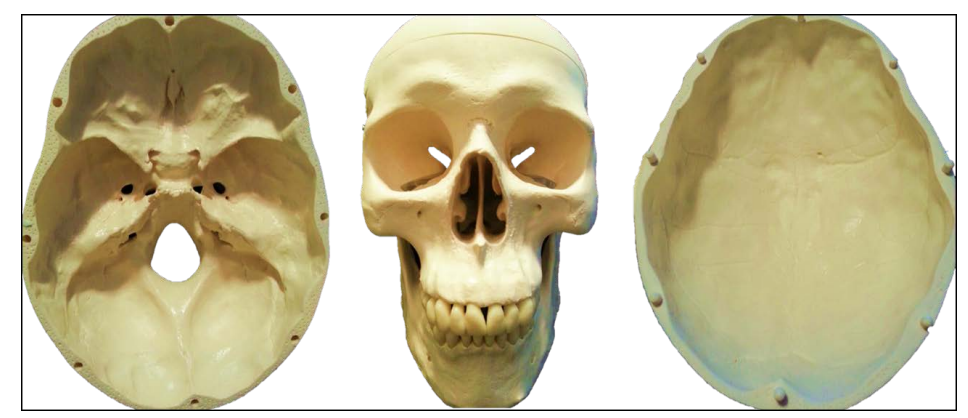

Figure 2. Replica human skull. Ventral (left) and dorsal (right) aspects of the internal cranial vault are shown. Realistic, non-symetrical cranial features are present includng processes, fossa, condyles, ridges, tuberosities, and sutures. 


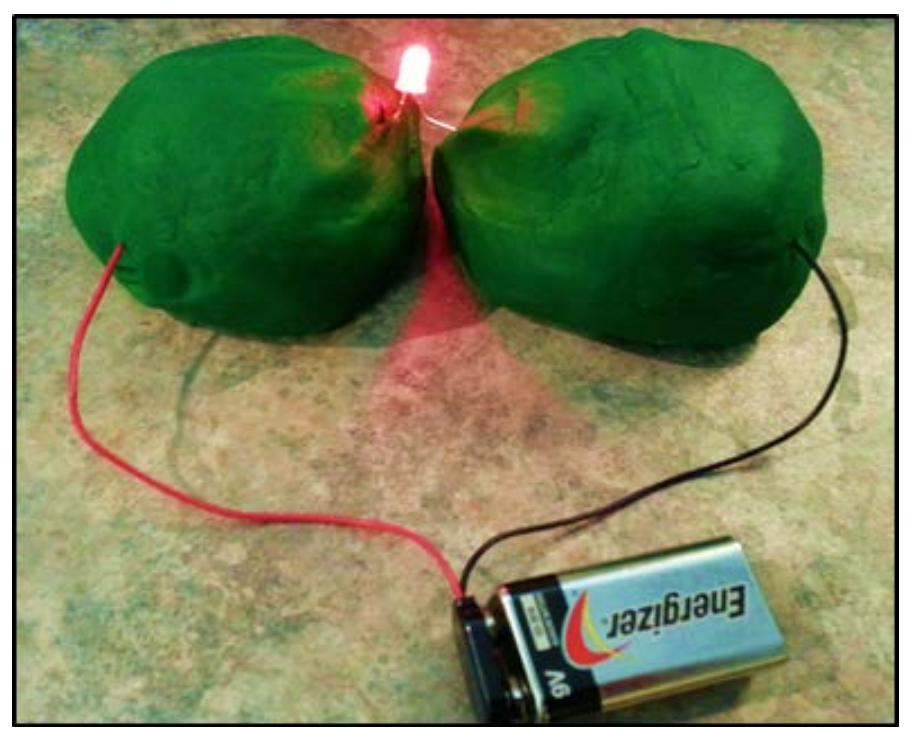

Figure 3. Squishy Circuits electroconductive dough coupled to a $9 \mathrm{~V}$ DC battery and a light-emitting diode (LED). This material lined the inner and outer aspects of a replica human skull to simulate the three-shell realistic head model.

Three dough recipes were prepared which differed only by concentration of the proton donor: 44 cc (low), 133 cc (medium), and 222 cc (high). Measurements were obtained in order to quantify the relative $\mathrm{pH}$ and total protons associated with each recipe as a function of this experimental manipulation. A pH sensor coupled to a Dr. DAQ data logger relayed information to a Lenovo laptop running PicoLog data acquisition software. The scan time of the $\mathrm{pH}$ meter was set to measure every $50 \mathrm{~ms}$, averaging values which were then recorded at a $1 \mathrm{~Hz}$ sampling rate. Each concentration of lemon juice was measured within the fixed volume of 237 cc of water within a glass beaker. $\mathrm{pH}$ measurements were obtained for 120 seconds and averaged across the trial to obtain the value associated with the recipe.

The high concentration condition was associated with a $\mathrm{pH}$ value of 2.47 whereas the medium and low concentration conditions were associated with $\mathrm{pH}$ values of 2.52 and 2.66 respectively. Hydrogen ion concentration (HIC) was then calculated $\left[\mathrm{H}^{+}\right]=10^{-\mathrm{pH}}$ which results in $\mathrm{M} / \mathrm{L}$. These values are then adjusted for volume and multiplied by Avagadro's number $\left(6.02 \times 10^{23} \mathrm{~mol} / \mathrm{M}\right)$. It was determined that the total hydrogen ions as a function of the donor were $\sim 10^{21}$ for the high concentration condition and $\sim 10^{20}$ for the medium and low concentration conditions. Although the difference was slightly less than a factor of ten, the total unit difference between the conditions exceeds $10^{20}$ proton units.

\subsection{Procedure}

QEEG measurements of ED baseline activity were obtained ( $\mathrm{n}=12$ ) with an associated sampling rate of $250 \mathrm{~Hz}$ and a notch-filter set to exclude frequency contributions between 45 and $75 \mathrm{~Hz}$. A Mitsar 19-channel QEEG system coupled to a HP ENVY laptop running Windows 8 served as the data logging equipment; however only four sensors (T3, T4, T5, and T6) were used due to the highly homogeneous activity of the ED across small spatial distances $(r=0.99, \mathrm{p}<0.001)$. These sensors correspond to those typically placed over areas that overlay the middle and posterior portions of the temporal lobe as per the 10 - 20 International System of Electrode Placement. Sensors were referenced to an average of sensor-clips positioned on either side of the ED.

Alternative geometrical configurations were measured within the three recipes which included the two conductive layers of the three-shell realistic head model (i.e. cortex and scalp) as well as two extra-cranial measurements. Sphere and disk shaped structures were formed with the ED using the same volumes of materials applied intra-skeletally $(180 \mathrm{cc})$. The surface area of the disk condition $\left(400 \mathrm{~cm}^{2}\right)$ was equivalent to that of the scalp layer of the three-shell head model.

Five $(n=5)$ eyes-open baseline QEEG profiles were randomly selected from a laboratory database containing hundreds of participants. These data served as baseline measures which could be statistically compared to the 
electrical activity associated with the ED. Although 19 sensors were involved in all human measurements, only sensors T3, T4, T5, and T6 were used for the current paradigm. Whereas human measurements were consistently within the $100-200 \mu \mathrm{V} / \mathrm{cm}$ range, ED activity was increased to approximately $200-300 \mu \mathrm{V} / \mathrm{cm}$.

\subsection{Computing Additional Variables}

Electrodes were clustered into left and right montages as well as a global montage incorporating all four sensors. QEEG measurements $\left(\mu \mathrm{V}^{2} / \mathrm{Hz}\right)$ were z-scored, spectral analyzed, and averaged across trials. Transformed variables were then re-combined to generate new factors. An intra-skeletal condition was generated by averaging (interfering) the spectral output of the scalp and cortex layers. An extra-skeletal condition was generated by the same process involving the disk and sphere configurations. Variables were generated for low, medium, and high HIC between and within the geometrical conditions.

\section{Results}

There was a moderate, positive correlation between intra-skeletal and human spectral densities within the narrow subset of gamma from $30-50 \mathrm{~Hz}, \mathrm{r}=0.60, \mathrm{p}<0.001$ corresponding to an effect size of 36\% (Figure 4). It was determined that the layer which contributed to this significant relationship was that of the cortex, $r=0.55, p$ $<0.001$. Extra-skeletal spectral densities (Figure 5) as well as the scalp layer of the three-shell model did not show this relationship.

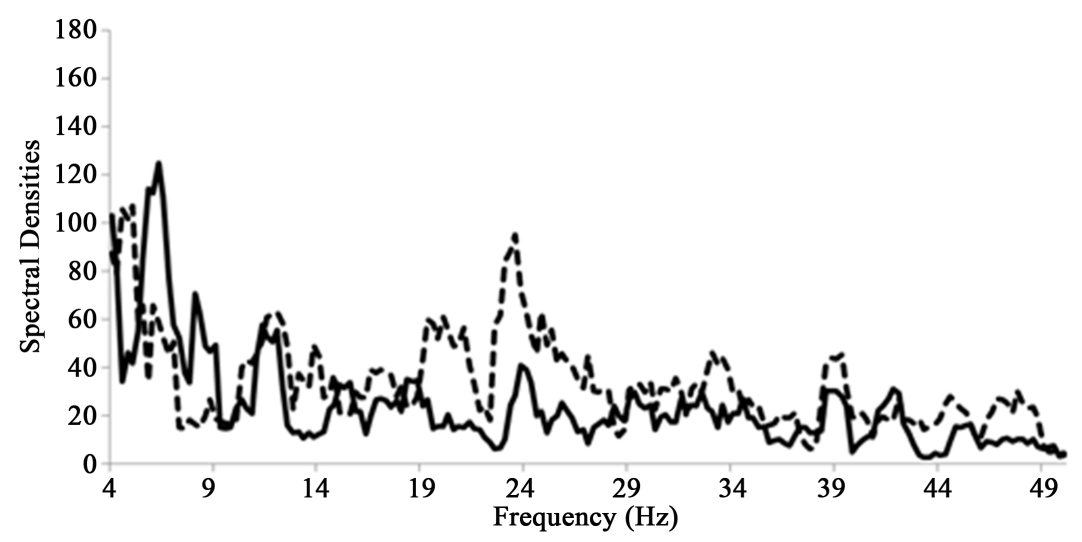

Figure 4. Spectral characteristics of z-transformed QEEG measurements of human baseline activity (dotted line) and intra-skeletal ED activity (solid line). The intra-skeletal condition is a composite of measurements obtained over the cortex and scalp layers (ED) bound to the replica skull which, together, forms the three-shell realistic head model.

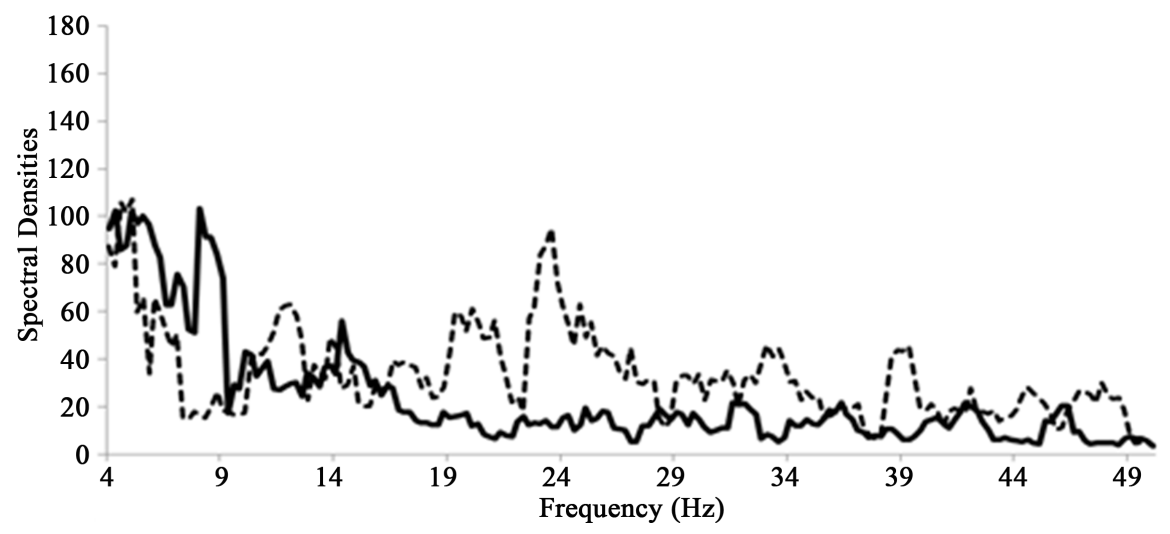

Figure 5. Spectral characteristics of z-transformed QEEG measurements of human baseline activity (dotted line) and extra-skeletal ED (solid line). The extra-skeletal condition consists of a composite of measurements obtained over a ball and a disk of ED placed on a flat surface. 
Correlational analyses performed for cases associated with Schumann frequency bands revealed a moderate, positive relationship between human and intra-skeletal spectral densities within the fundamental of $\sim 8 \mathrm{~Hz}$ ( $\mathrm{r}=$ $0.59, \mathrm{p}<0.005$ ) as well as the first harmonic or $14 \mathrm{~Hz}, \mathrm{r}=0.53, \mathrm{p}<0.01$. When performing the analysis separately upon the two conductive layers of the three-shell model, spectral densities of the cortex moderately correlated with human spectral densities for the fundamental Schumann frequency $(r=0.59, p<0.005)$; however, the scalp layer was not significantly correlated $(r=0.30, p>0.05)$. Extra-skeletal spectral densities did not correlate with those of the human sample within the first three frequency bands; however, within the frequency band corresponding to the fourth harmonic $(33 \mathrm{~Hz})$, extra-skeletal and human spectral densities were moderately correlated, $\mathrm{r}=0.58, \mathrm{p}<0.005$. The cortex layer spectral densities were also correlated with human spectral densities within the frequency band corresponding to the fourth harmonic $(r=0.52, p=0.01)$. This relationship was not observed for the spectral densities derived from the electrical activity of the scalp layer alone or in combination with the cortex layer.

ED containing high $(\mathrm{r}=0.50, \mathrm{p}<0.001)$ and medium $(\mathrm{r}=0.48, \mathrm{p}<0.001)$ proton concentrations showed moderate, positive correlations with human spectral densities between $30-50 \mathrm{~Hz}$ which were not apparent for the low concentration conditions, $\mathrm{r}=-0.01, \mathrm{p}>0.05$. The observed correlations were not significantly different, $\mathrm{zF}=-0.16, \mathrm{p}>0.05$. Figure 6 shows that the spectral characteristics emergent of the high hydrogen ion concentration condition (black) displays peaks of increased oscillatory activity within frequency bands associated with the Schumann resonance. The low hydrogen ion condition (red) displayed relatively equal contributions of power under most frequency bands (i.e. increased noise) with the exception of the $10-13 \mathrm{~Hz}$ range.

Examining the simulated cortex layer only for each proton concentration condition for the typical waking, human electroencephalographic bandwidth $(4-50 \mathrm{~Hz})$ revealed a moderate positive correlation between spectral densities of the high hydrogen ion concentration cortex condition and human spectral densities $(r=0.60, p<$ 0.001). Figure 7 shows spectral densities associated with the high hydrogen ion concentration cortex condition with human spectral densities. This relationship, although observed, was significantly diminished for the low and medium hydrogen ion concentration cortex conditions, $\mathrm{zF}>1.96, \mathrm{p}<0.01$. There was also a significant difference between the correlation coefficient illustrated in Figure 8 and the significant but weak correlations between human spectral densities and those of the ED sphere $(\mathrm{r}=0.38, \mathrm{p}<0.001)$ and ED disk $(\mathrm{r}=0.41, \mathrm{p}<$ 0.001), $\mathrm{zF}>1.96, \mathrm{p}<0.001$. Figure 8 and Figure 9 show the ED that were shaped as a sphere or a disk paired with human spectral densities. These results suggest that the intrinsic oscillatory activity of the cortex layer within the "high" hydrogen ion concentration condition was more strongly associated with human spectral densities than any other condition.

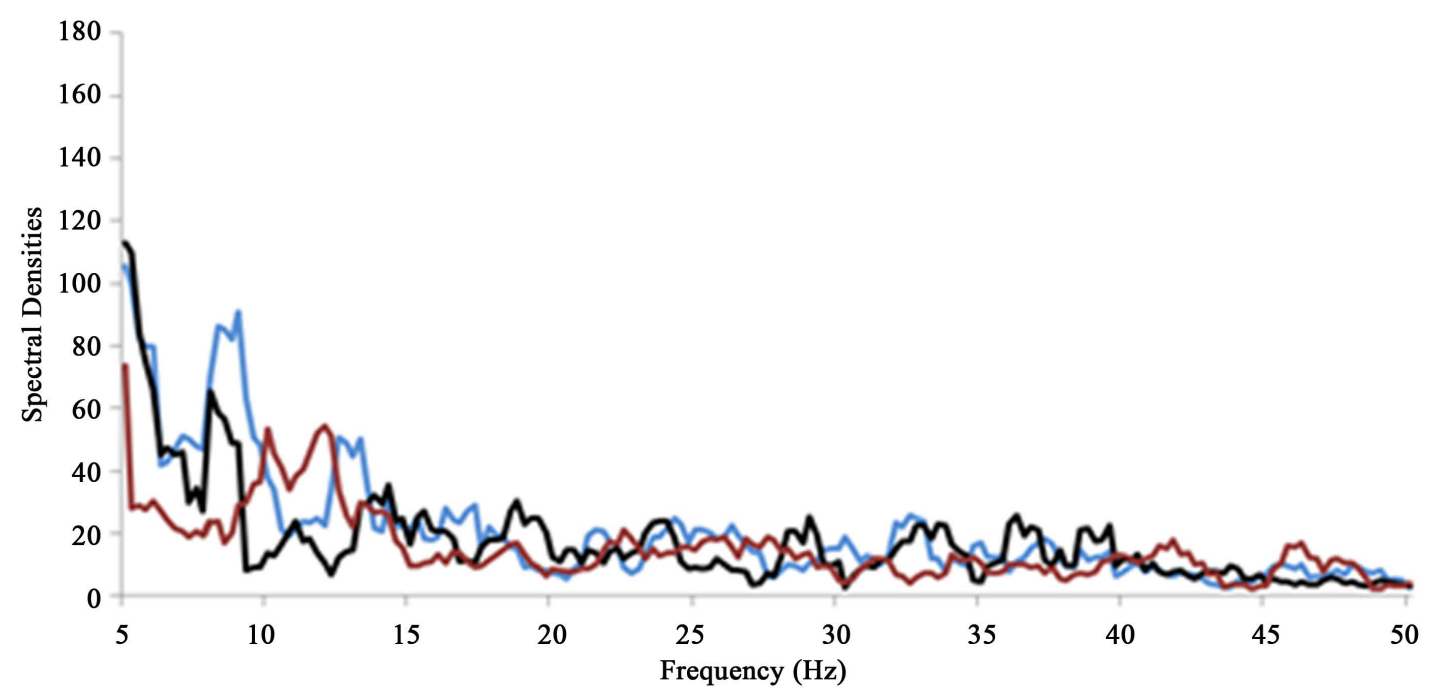

Figure 6. Spectral characteristics of ED recipes with high (black), medium (blue), and low (red) hydrogen ion concentrations. ED containing high hydrogen ion concentrations displays peaks typical of the Schumann resonance including $8 \mathrm{~Hz}, 14 \mathrm{~Hz}$, and $\sim 19 \mathrm{~Hz}$. A phase-shifted series of subsequent peaks separated by a relatively constant interval are present. 


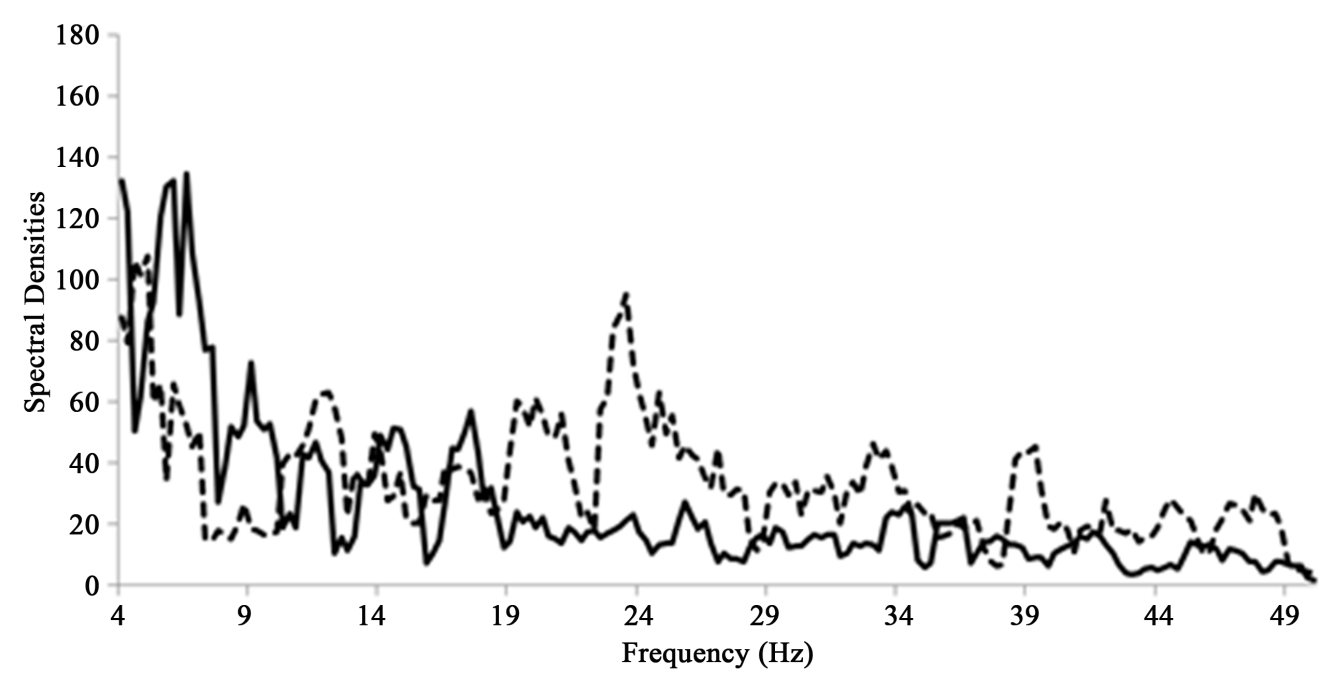

Figure 7. Spectral characteristics of z-transformed QEEG measurements of human baseline activity (dotted line) and activity associated with the cortex layer of the three-shell head model within the high hydrogen ion concentration condition (solid line).

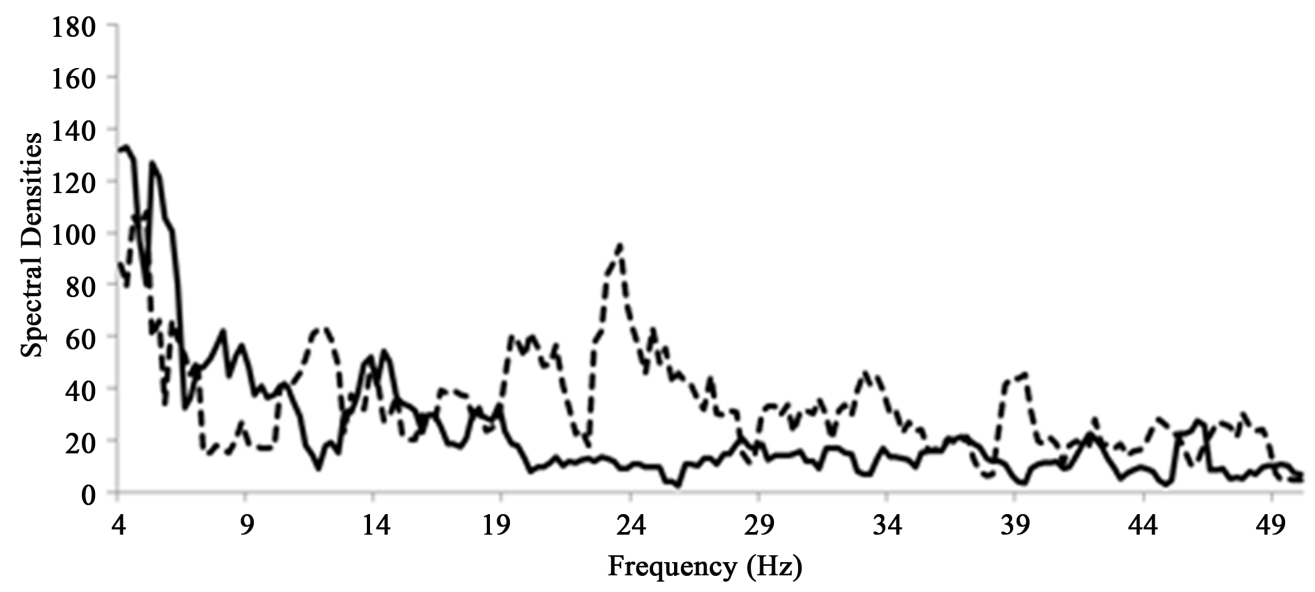

Figure 8. Spectral characteristics of z-transformed QEEG measurements of human baseline activity (dotted line) and activity associated with ED as a sphere placed on a flat surface (solid line).

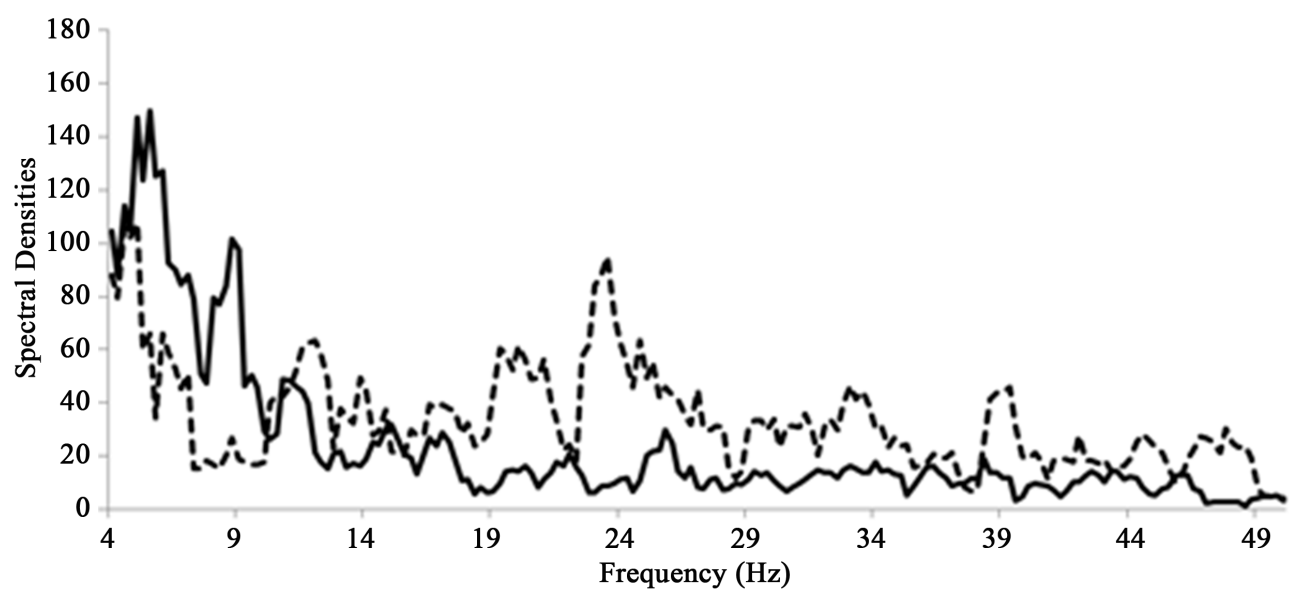

Figure 9. Spectral characteristics of z-transformed QEEG measurements of human baseline activity (dotted line) and activity associated with ED as a disk placed on a flat surface (solid line). 


\section{Discussion}

The "spontaneous" spectral densities for an electro-conductive medium (ED) occupying the approximate volume of the human cerebral cortices and containing "high" ( $\mathrm{pH}=2.47)$ or "medium" ( $\mathrm{pH}=2.52$ ) proton concentrations showed a significant overlap with human spectral densities which were not present for the "low" ( $\mathrm{pH}$ = 2.66) concentration condition. Other geometries, such as a flat disk or solid spherical shape did not display these significant cross-correlations with actual human cerebral cortical spectral densities.

The fact that a critical number of protons were required strongly indicates the moderate strength correlation between spectral densities observed from passive measurement of the ED and those of actual human brains was not an artefact of the QEEG or our procedures. If procedural artefacts were present then the moderate strength correlations should have been evident when all concentrations of protons were employed. However, the significant correlations were only evident for the $\mathrm{pH}$ values $<2.53$.

The direct application of these results and this model to actual brain function may appear to be limited by the marked discrepancy in $\mathrm{pH}$. The typical levels of $\mathrm{pH}$ in intracellular space during neuronal activity are around 7.0 with shifts of about $0.2 \mathrm{pH}$ units. On the other hand the $\mathrm{pH}$ levels within our ED simulation was much more acidic, about $\mathrm{pH}=2.5$. The most parsimonious solution to this apparent discrepancy involves the numbers of $\mathrm{H}^{+}$ within the functional volume. The ionic source of the resting membrane potential for plasma cell membranes for soma around $10 \mu \mathrm{m}$ in width is about the width of a Bohr radius or $5.6 \times 10^{-9} \mathrm{~cm}$ [31]. The shell (assuming a sphere) occupied by this additional volume is $1.8 \times 10^{-14} \mathrm{cc}$. The numbers of $\mathrm{H}^{+}\left(\mathrm{M}=10^{-\mathrm{pH}}\right)$ within this volume with a $\mathrm{pH}=2.5$ would be $3.16 \times 10^{-3} \mathrm{M}$ multiplied by $9.8 \times 10^{-16}$ cc $\left(1.8 \times 10^{-14}\right.$ cc divided by $18 \mathrm{cc}$ per $\left.\mathrm{M}\right)$ or $31 \times 10^{-19} \mathrm{M}$. When multiplied by Avogadro's constant, the numbers of $\mathrm{H}^{+}$would be about $1.9 \times 10^{6}$ molecules.

On the other hand, the volume occupied by a shell that extends $1 \mu \mathrm{m}$ from the cell, where experimental $\mathrm{pH}$ levels are typically taken, would involve a volume of $3.8 \times 10^{-10}$ cc or $2.1 \times 10^{-11} \mathrm{cc}\left(3.82 \times 10^{-10}\right.$ divided by 18 cc per $\mathrm{M}$ ). For a $\mathrm{pH}$ of 7 the numbers of $\mathrm{H}^{+}$would be the same order of magnitude (about $10^{6}$ ) as that calculated for the more acidic $\mathrm{pH}$ within a single layer of charges. Interesting this is the estimated numbers of charges required to maintain the resting plasma membrane potential [7]. This convergence suggests that the functional concentration of $\mathrm{H}^{+}$along the proximal surface that markedly influences the resting cell membrane potential is much more acidic than typically assumed and more typical of what we employed in the ED model. The primary difference between the ED "cerebral cortex" and the natural human cerebral cortex is that the thin shell of protons would line an extraordinarily complex surface structure along the membrane of neuronal soma, dendrites and axons within the $\sim 3 \mathrm{~mm}$ thick cortical shell.

The convergent of numbers of $\mathrm{H}^{+}$for the measured neuronal environment when the single layer of charge along the plasma cell membrane is considered has significant implication for mechanisms of consciousness. According to Bordag et al. [32] only the surface layer of atoms or charges can be considered a continuum. It is this continuum that interacts with an electromagnetic field. The Casimir effect, which emerges in space-times with nontrivial topology (such the cerebral cortex) is the interaction between a pair of parallel conducting plates due the disturbance of the vacuum of the electromagnetic field. Bordag et al. [32] describes this process as a pure quantum effect. This connects the Casimir effect to the existence of Zero Point Fluctuations and the forces coupled with Gravity [33].

That gravity is relevant to consciousness is illustrated by the solution for the potential energy for a human brain's mass. Gravitational energy is classically defined as $\mathrm{G} \cdot \mathrm{kg}^{2} \cdot \mathrm{m}^{-1}$, where $\mathrm{G}$ is the Newtonian Gravitational Constant $\left(6.67 \times 10^{-11} \mathrm{~m}^{3} \cdot \mathrm{kg}^{-1} \cdot \mathrm{s}^{-2}\right)$, $\mathrm{kg}$ is the mass of the human brain (assume $1.5 \mathrm{~kg}$ ) and $\mathrm{m}$ is the average length (assume $12 \mathrm{~cm}$ ). The resulting energy is $1.25 \times 10^{-9} \mathrm{~J}$. Assuming an increment of energy of $\sim 2 \times 10^{-20} \mathrm{~J}$ for the effects of an action potential $(120 \mathrm{mV})$ upon a unit charge $\left(1.6 \times 10^{-19} \mathrm{~A} \cdot \mathrm{s}\right)$ with the average frequency of $10 \mathrm{~Hz}$, the gravitational energy would have the potential to be equivalent to about $10^{10}$ neurons, which is with the order of magnitude of functioning cells within the human cerebral cortices [7].

For the entire volume, we suggest the value of $\sim 10^{21}$ units represents a critical threshold-a necessary condition-at which point Schumann-type oscillations are present in the spectral profile of the ED. Assuming the hydron isotope of a proton $\left(\mathrm{H}^{+}\right)$with an associated mass of $1.67 \times 10^{-7} \mathrm{~kg}$, the mass equivalency of $\sim 10^{21}$ protons is $1.5 \times 10^{-6} \mathrm{~kg}$. Because the typical cell is 1 nanogram or $10^{-12} \mathrm{~kg}$, the number of cells associated with this mass-equivalency of the hydron threshold is $\sim 1.5 \times 10^{6}$ cells or between 1 and 2 million. This is the approximate number of cells which constitute the hippocampus [34], a body which is linked to Schumann activity [20]. The value of $10^{6}-10^{7}$ neurons also constitutes the typical discrete cluster of cells associated with a "thought" [35]. 
We pursued the idea that hydrogen ion matrices composed of a minimum of $\sim 10^{21}$ units are intrinsic receptive structures associated with the Schumann resonance. The volume of the human neocortex is $\sim 5 \times 10^{2} \mathrm{ml}$ on average with considerable variability within and between sexes [36]. Converting to grams, $\sim 60 \%$ of the mass is water or $3 \times 10^{2} \mathrm{~g}$. This mass can be further reduced to its hydrogen component which is $11 \%$ by proportional mass or a total of $33 \mathrm{~g}$. If the molar mass of $\mathrm{H}_{2} \mathrm{O}$ is $18 \mathrm{~g} / \mathrm{M}$, this volume represents $1.83 \mathrm{M}$ that, when multiplied by Avagadro's number gives $1 \times 10^{24}$ units within the human cerebral cortex of available hydrogen to dissociate. If the ED and the cerebral cortex are responding in the same way due to microstructural similarity in hydron content, the suggestion would be that a critical threshold of hydron units is needed to resonate with the ionosphere and express the Schumann pattern.

Spectral densities associated with the intra-skeletal condition optimally correlated with human baseline brain activity. This was primarily due to the cortex layer of the model, which suggests an intrinsic role of the shape of the skull in the representation of Schumann patterns in QEEG profiles. Nunez [37] has presented works which demonstrate that the bulk velocity of action potentials within the cerebral cortices $\left(4.5 \mathrm{~m} \cdot \mathrm{s}^{-1}\right)$ applied over a skull with a $60 \mathrm{~cm}$ circumference would generate a standing wave of approximately $7.5 \mathrm{~Hz}$-value approximating the fundamental Schumann resonance frequency. A recent quantitative analysis of Earth-Brain interactions has revealed multiple convergences which suggest a profound relationship between the two structures [5]. Where neurons contained within the skull provide structure, the chemical and geometrical orientation of the space surrounding neurons are the primary interfaces for phenomena which precede the action potential. In the absence of neurons, the lattice of the dough might provide a similar network of structures along which signals might propagate in the presence of an external electromagnetic stimulus.

In this study, synchronous gamma oscillations within the $40 \mathrm{~Hz}$ range were measured in a three-shell realistic head model which was constructed to simulate the basic organization of the human cortex, scalp, and skull. As a neural correlate of consciousness, the expression of synchronous gamma in an intra-skeletal organization of ED does not indicate that the experimental apparatus was conscious. The only suggestion here is that a neural correlate of consciousness which operated within parameters similar to that observed in humans was disembodied from any neuronal substrate in a geometrical and chemical approximation of the components of the brain measured by the QEEG. These distinct, physiologically-patterned oscillations were not present in the same material shaped as a sphere or disk placed outside of the skull.

One theoretical solution involves a transmission theory of brain activity whereby an external signal is filtered by the brain and intermediate tissues [38]. Coherent oscillations across spaces which do not accommodate typical structural connections would be accommodated as receptive fields and would respond similarly to the application of the external stimulus. These responses, although simultaneous and independent, would be coherent and perhaps vulnerable to intra-cerebral resonance phenomena. Synchronous activity across brain-space is intrinsically linked to the binding problem [39]. If the three-shell model is operating as an antenna and receiving signals from the environment, this project lends experimental validity to the metaphor of consciousness and other brain functions as transmitted information.

Kuhn [40] argued that paradigm shifts are necessary and are brought about by a growing sense that existing models, albeit once optimally useful in pursuit of further knowledge, have malfunctioned in some fundamental way. In order to accommodate the observations that consciousness has avoided the traditional neuroscientific line of inquiry involving localization of function in brain-space, the idea of extra-cerebral signals and the information contained within them must be seriously considered as a potential source for the neural correlates of consciousness.

\section{Conclusion}

Here we present results indicating oscillatory electrical activity in a non-human model of the human head displays spectral densities that correlate with those of human subjects obtained over the scalp using QEEG technology. Quantification suggests a central role of the proton in relation to the physical dimensions of the cell. Further experiments are required which would parcel out the environmental contributions to the neural correlates of consciousness and other functions typically ascribed to the brain. In particular, the capacity for this preparation to show simulations of "memory", that is the representation of previous stimuli no longer present, and learning, the more or less permanent change in responsiveness due to experience, should be demonstrable. 


\section{Acknowledgements}

The authors would like to thank Professor Kevin Saroka for his insights and technical assistance.

\section{References}

[1] Deamer, D. (2011) Consciousness and Intelligence in Mammals: Complexity Thresholds. Journal of Cosmology, 14.

[2] McIntosh, A.R. (2000) Towards a Network Theory of Cognition. Neural Networks, 13, 861-870. http://dx.doi.org/10.1016/S0893-6080(00)00059-9

[3] Alberts, B., Bray, D., Lewis, J., Raff, M., Roberts, K. and Watson, J.D. (1994) Molecular Biology of the Cell. Garland, New York, 50-56.

[4] Persinger, M.A. and Dotta, B.T. (2011) Temporal Patterns of Photon Emissions Can Be Stored and Retrieved Several Days Later from the "Same Space”: Experimental and Quantitative Evidence. NeuroQuantology, 9, 605-613. http://dx.doi.org/10.14704/nq.2011.9.4.467

[5] Persinger, M.A. (2014) Quantitative Convergence between Physical-Chemical Constants of the Proton and the Properties of Water: Implications for Sequestered Magnetic Fields and a Universal Quantity. International Letters of Chemistry, Physics and Astronomy, 1, 1-10.

[6] Decoursey, T.E. (2003) Voltage-Gated Proton Channels and Other Proton Transfer Pathways. Physiological Reviews, 83, 475-579.

[7] Persinger, M.A. (2010) $10^{-20}$ Joules as a Neuromolecular Quantum in Medicinal Chemistry: An Alternative Approach to Myriad Molecular Pathways? Current Medicinal Chemistry, 17, 3094-3098. http://dx.doi.org/10.2174/092986710791959701

[8] Bevan, S. (1998) Proton-Gated Ion Channels in Neurons. In: Kaila, K. and Ransom, B.R., Eds., pH and Brain Function, Wiley-Liss, New York, 447-476.

[9] Highstein, S.M., Holstein, G.R., Mann, M.A. and Rabbitt, R.D. (2014) Evidence That Protons Act as Aeurotransmitters at Vestibular Hair Cell-Calyx Afferent Synapses. Proceedings of the National Academy of Sciences, 111, 5421-5426. http://dx.doi.org/10.1073/pnas.1319561111

[10] Murugan, N.J., Karbowski, L.M. and Persinger, M.A. (2014) Serial pH Increments ( 20 to 40 Milliseconds) in Water during Exposures to Weak, Physiologically Patterned Magnetic Fields: Implications for Consciousness. Water, 6, 4560.

[11] Llinas, R. and Ribary, U. (1993) Coherent 40-Hz Oscillation Characterizes Dream State in Humans. Proceedings of the National Academy of Sciences of the United States of America, 90, 2078-2081. http://dx.doi.org/10.1073/pnas.90.5.2078

[12] Persinger, M.A. (2013) Billions of Human Brains Immersed within a Shared Geomagnetic Field: Quantitative Solutions and Implications for Future Adaptations. Open Biology Journal, 6, 8-13. http://dx.doi.org/10.2174/1874196701306010008

[13] Crick, F. and Koch, C. (1990) Towards a Neurobiological Theory of Consciousness. Seminars in the Neurosciences, 2, 263-275.

[14] Bear, M.F. (1996) A Synaptic Basis for Memory Storage in the Cerebral Cortex. Proceedings of the National Academy of Sciences of the United States of America, 93, 13453-13459. http://dx.doi.org/10.1073/pnas.93.24.13453

[15] Schlegel, K. and Füllekrug, M. (2002) 50 Years of Schumann Resonance. Physik in Unserer Zeit, 33, 256-264.

[16] Schumann, W.O. (1952) On the Free Oscillations of a Conducting Sphere Which Is Surrounded by an Air Layer and an Ionosphere Shell. Z. Naturforschaft, 7A, 149-154.

[17] König, H. (1971) Meteorological Cycles: Biological Effects of Extremely Low Frequency Electrical Phenomena in the Atmosphere. Biological Rhythm Research, 2, 317-323.

[18] Cherry, N. (2002) Schumann Resonances, a Plausible Biophysical Mechanism for the Human Health Effects of Solar. Natural Hazards, 26, 279-331. http://dx.doi.org/10.1023/A:1015637127504

[19] Cherry, N. (2003) Human Intelligence: The Brain, an Electromagnetic System Synchronised by the Schumann Resonance Signal. Medical Hypotheses, 60, 843-844. http://dx.doi.org/10.1016/S0306-9877(03)00027-6

[20] Persinger, M.A. (2008) On the Possible Representation of the Electromagnetic Equivalents of All Human Memory within the Earth's Magnetic Field: Implications for Theoretical Biology. Theoretical Biology Insights, 1, 3-11.

[21] Alonso, A. and Klink, R. (1993) Differential Electroresponsiveness of Stellate and Pyramidal-Like Cells of Medial Entorhinal Cortex Layer II. Journal of Neurophysiology, 70, 128-143.

[22] Persinger, M.A. (1976) Day Time Wheel Running Activity in Laboratory Rats Following Geomagnetic Event of 5-6 
July 1974. International Journal of Biometeorology, 20, 19-22. http://dx.doi.org/10.1007/BF01553167

[23] Stewart, L.S. and Persinger, M.A. (2000) Pretraining Exposure to Physiologically Patterned Electromagnetic Stimulation Attenuates Fear-Conditioned Analgesia. International Journal of Neuroscience, 100, 91-98. http://dx.doi.org/10.3109/00207450008999679

[24] Cifra, M., Fields, J.Z. and Farhadi, A. (2011) Electromagnetic Cellular Interactions. Progress in Biophysics and Molecular Biology, 105, 223-246. http://dx.doi.org/10.1016/j.pbiomolbio.2010.07.003

[25] Gang, N., St-Pierre, L.S. and Persinger, M.A. (2012) Water Dynamics Following Treatment by One Hour 0.16 Tesla Static Magnetic Fields Depend on Exposure. Water, 3, 122-131.

[26] Dotta, B.T., Buckner, C.A., Lafrenie, R.M. and Persinger, M.A. (2011) Photon Emissions from Human Brain and Cell Culture Exposed to Distally Rotating Magnetic Fields Shared by Separate Light-Stimulated Brains and Cells. Brain Research, 1388, 77-88. http://dx.doi.org/10.1016/j.brainres.2011.03.001

[27] Beg, A.A., Ernstrom, G.G., Nix, P., Davis, M.W. and Jorgensen, E.M. (2008) Protons Act as a Transmitter for Muscle Contraction in C. elegans. Cell, 132, 149-160. http://dx.doi.org/10.1016/j.cell.2007.10.058

[28] Burke, R.C. and Persinger, M.A. (2013) Convergent Quantitative Solutions Indicating the Human Hippocampus as a Singularity and Access to Cosmological Consciousness. NeuroQuantology, 11, 1-7. http://dx.doi.org/10.14704/nq.2013.11.1.646

[29] Berg, P. and Scherg, M. (1994) A Fast Method for Forward Computation of Multiple-Shell Spherical Head Models. Electroencephalography and Clinical Neurophysiology, 90, 58-64. http://dx.doi.org/10.1016/0013-4694(94)90113-9

[30] Ermer, J.J., Mosher, J.C., Baillet, S. and Leahy, R.M. (2001) Rapidly Recomputable EEG Forward Models for Realistic Head Shapes. Physics in Medicine and Biology, 46, 1265-1281.

[31] Persinger, M.A. (2012) Brain Electromagnetic Activity and Lightning: Potentially Congruent Scale-Invariant Quantitative Properties. Frontiers in Integrative Neuroscience, 6, 1-7.

[32] Bordag, M., Mohideen, U. and Mostepanenko, V.M. (2001) New Developments in the Casimir Effect. Physics Reports, 353, 1-205. http://dx.doi.org/10.1016/S0370-1573(01)00015-1

[33] Puthoff, H.E. (1989) Source of Vacuum Electromagnetic Zero-Point Energy. Physical Review A, 40, 4857. http://dx.doi.org/10.1103/PhysRevA.40.4857

[34] West, M.J. and Gundersen, H.J.G. (1990) Unbiased Stereological Estimation of the Number of Neurons in the Human Hippocampus. Journal of Comparative Neurology, 296, 1-22. http://dx.doi.org/10.1002/cne.902960102

[35] Levy, I., Hasson, U. and Malach, R. (2004) One Picture Is Worth at Least a Million Neurons. Current Biology, 14, 996-1001. http://dx.doi.org/10.1016/j.cub.2004.05.045

[36] Pakkenberg, B. and Gundersen, H.J.G. (1997) Neocortical Neuron Number in Humans: Effect of Sex and Age. Journal of Comparative Neurology, 384, 312-320.

[37] Nunez, P.L. (1995) Neocortical Dynamics and Human EEG Rhythms. Oxford University Press, New York.

[38] James, W. (1898) Human Immortality: Two Supposed Objections to the Doctrine. Houghton Mifflin, Boston.

[39] Meador, K.J., Ray, P.G., Echauz, J.R., Loring, D.W. and Vachtsevanos, G.J. (2002) Gamma Coherence and Conscious Perception. Neurology, 59, 847-854. http://dx.doi.org/10.1212/WNL.59.6.847

[40] Kuhn, T.S. (1962) The Structure of Scientific Revolutions. University of Chicago Press, Chicago, 1-15. 
Scientific Research Publishing (SCIRP) is one of the largest Open Access journal publishers. It is currently publishing more than 200 open access, online, peer-reviewed journals covering a wide range of academic disciplines. SCIRP serves the worldwide academic communities and contributes to the progress and application of science with its publication.

Other selected journals from SCIRP are listed as below. Submit your manuscript to us via either submit@scirp.org or Online Submission Portal.
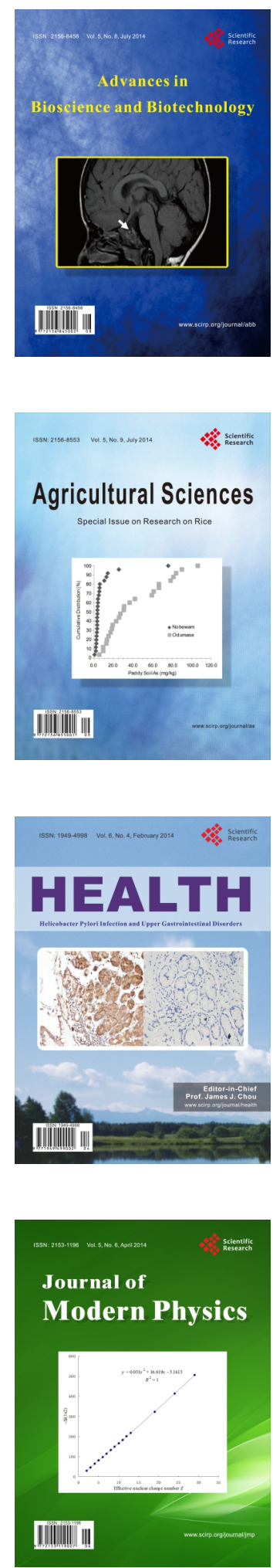
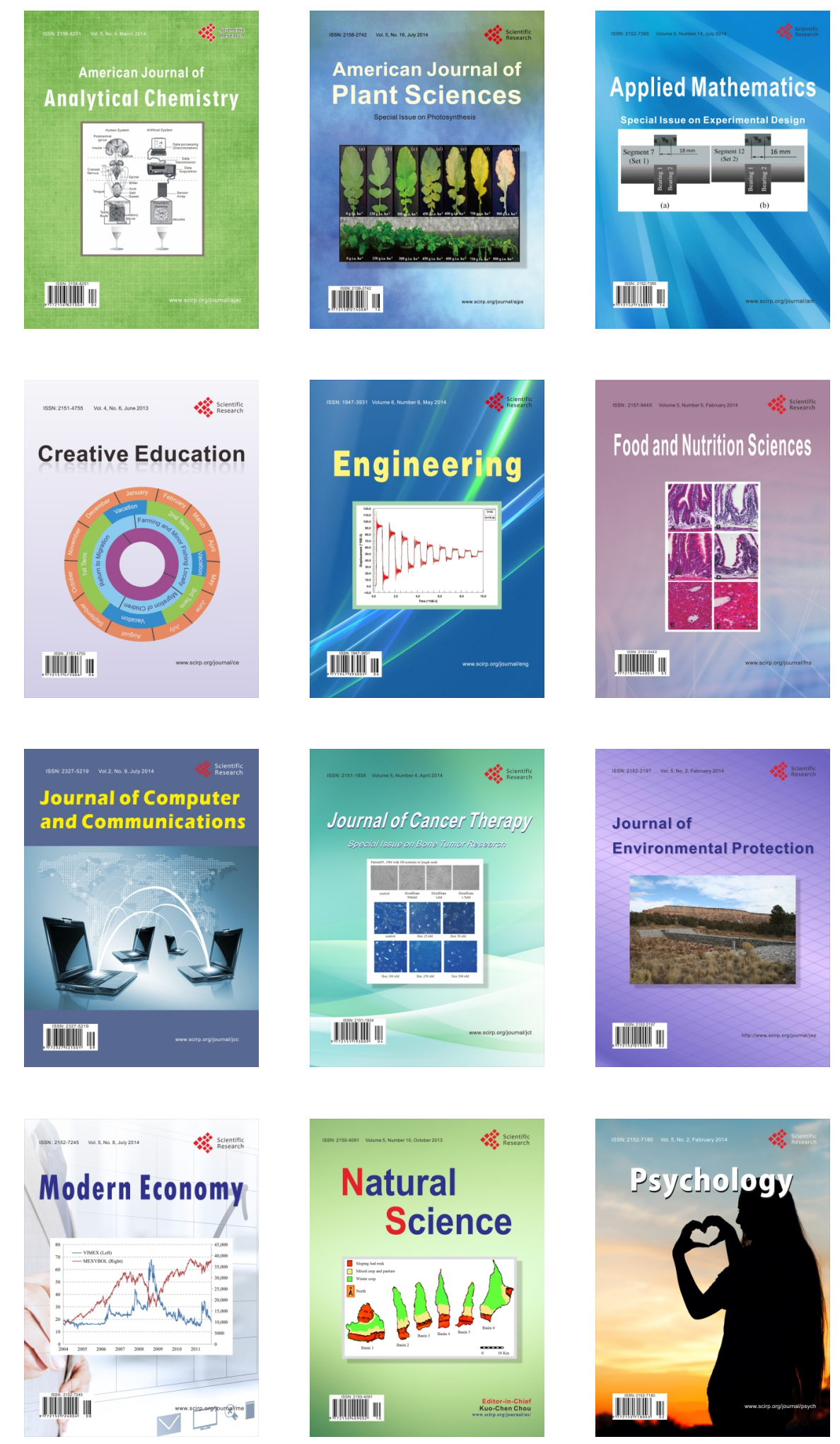